Mots. Les langages du politique

\title{
Entre espace urbain et espace numérique. La mobilisation immobile des Sentinelles contre le mariage homosexuel
}

Between urban space and digital space. The motionless mobilisation of the Sentinelles against same-sex marriage

Entre el espacio urbano y el espacio numérico. La mobilización inmóvil de las Sentinelles contra el matrimonio homosexual

\section{Elisa Raschini}

\section{OpenEdition \\ Journals}

Édition électronique

URL : https://journals.openedition.org/mots/22203

DOI : $10.4000 /$ mots.22203

ISSN : 1960-6001

Éditeur

ENS Éditions

Édition imprimée

Date de publication : 9 mai 2016

Pagination : 31-47

ISBN : 978-2-84788-793-8

ISSN : 0243-6450

\section{Référence électronique}

Elisa Raschini, «Entre espace urbain et espace numérique. La mobilisation immobile des Sentinelles contre le mariage homosexuel », Mots. Les langages du politique [En ligne], 110 | 2016, mis en ligne le 09 mai 2018, consulté le 24 avril 2022. URL : http://journals.openedition.org/mots/22203 ; DOI : https://doi.org/10.4000/mots.22203 


\section{Entre espace urbain et espace numérique. La mobilisation immobile des Sentinelles contre le mariage homosexuel}

Cet article s'interroge sur la signification d'un geste qui, par son absence de mouvement, ne semble pas en être un : se tenir debout, immobile. Telle est l'action choisie par les Sentinelles pour se mobiliser en France depuis le 24 juin 2013. À compter de ce jour, par le biais des réseaux sociaux sur l'Internet, une mobilisation issue du collectif de la Manif pour tous (désormais LMPT) contre la loi ouvrant le mariage aux couples homosexuels s'est créée, dont la performance veut que chacun reste debout, immobile, en silence, espacé des autres, sans signe distinctif, devant des lieux symboliques de pouvoir (Place Vendôme à Paris, par exemple. Voir figure 1 page suivante).

Se caractérisant par son immobilité, cette posture ne semble pas respecter le critère perceptif le plus saillant de la définition lexicographique du geste, «mouvement du corps », selon Le Petit Robert. Or, le critère cinétique, tout en étant pertinent, ne suffit pas, à lui seul, à définir la catégorie du geste. Il suffit en effet d'affirmer, avec l'ethnologue Bernard Kœchlin, que la posture est un geste «à cinématique nulle» (Kœchlin, 1991, p. 163) pour montrer le caractère aussi intuitif que fuyant de la notion de geste. B. Kœchlin nous offre aussi, grâce à sa notion de gestuelle, la possibilité de dépasser le seul critère cinétique : "par son pouvoir de matérialisation, par une mise en espace, ou une mise en volume », la gestuelle fonctionne comme "un passage obligé de tout processus de concrétisation d'un concept, dans toute transmission du sens, dans toute pratique ou technique issue d'une chaîne opératoire préfigurée» (Kœchlin, 1991, p. 170).

Le geste - y compris la posture - est ici pensé comme la «mise en volume» d'une signification. Nous nous interrogerons ainsi sur la signification de ce geste qu'est la posture des Sentinelles, telle qu'elle émerge du rapport entre «mise en volume» et dimension discursive. "Si quelque chose se passe, il ne suffit pas en effet de le décrire, mais il y a lieu de s'interroger sur ce que cela implique pour les acteurs ou les témoins de l’événement » (Londei et al., 2013, p. 13). Si la 
Figure 1. Des Sentinelles devant le ministère de la Justice à Paris. (c) Elisa Raschini.

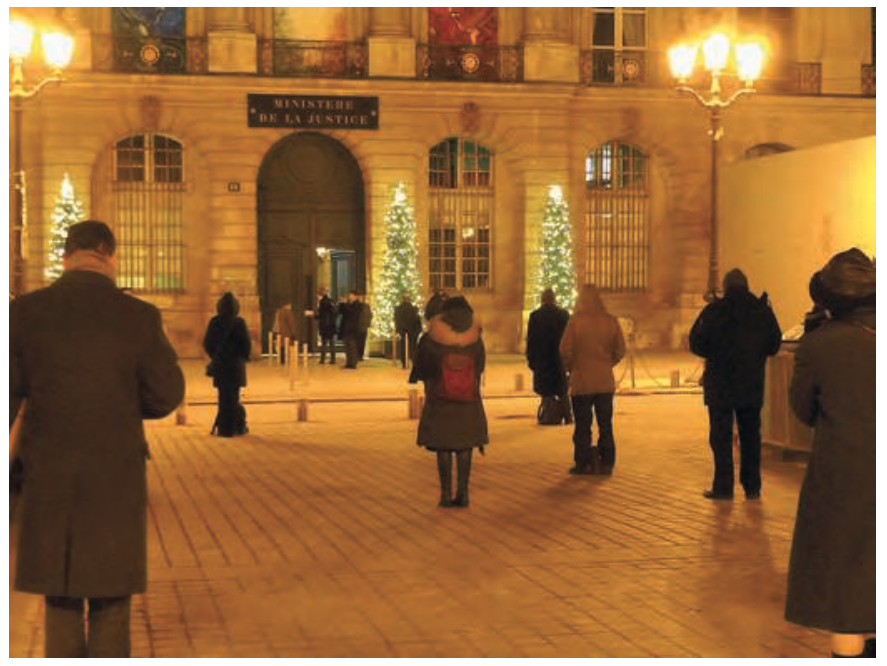

posture debout, «à l'origine de la plupart de nos gestes » (Carénini, 1991, p. 75), peut être considérée comme universelle et, en un sens, pré-culturelle, ce qui doit alors être questionné, lorsqu'on s’interroge sur sa signification, est moins sa description que le rapport entre ce qui est montré sur la place et ce qui est dit en discours : l'articulation entre mise en scène et mise en mots.

Nous aborderons cette question sous l'angle sémantico-discursif en tentant de mettre en lumière, par une démarche analytique et heuristique, des cheminements de sens entre les différentes productions langagières au niveau lexical, syntaxique, énonciatif, mémoriel. Nous tenterons de reconstituer certains de ces trajets, en diachronie et en synchronie, pour contribuer à la compréhension de la «vaste narration » - selon l'expression de Jacques Guilhaumou (2006, p.121) - qui concerne, crée et déploie en discours un objet en tant qu'événement discursif : en l'occurrence, celui de la mise en scène des Sentinelles.

Nous étudierons le discours des Sentinelles ou de ceux qui, en parlant d'elles, se réclament des mêmes positionnements idéologiques. Les Sentinelles utilisent des tweets, elles publient des posts depuis leur page Facebook et des textes sur des sites internet. Alors que les tweets sont plutôt mobilisés pour le témoignage et le commentaire synchronique et collectif des faits, les posts sont plutôt choisis pour la diffusion d'informations, de manifestes idéologiques et de chartes régulatrices. Différentes quant au caractère plus ou moins horizontal et participatif des échanges, ces productions langagières sont réunies par leur lien constitutif à la dimension numérique et technologique dans laquelle elles se réalisent. Leur technodiscursivité (voir Paveau, 2013a) n'af- 
fecte pas seulement le langage (notamment par la présence de l'hypertextualité des technomots et des liens cliquables) : elle affecte aussi, d'un autre point de vue, la modalité dont les plus récents mouvements sociaux envisagent et organisent leur propre action (voir Castells, 2012). La technodiscursivité permet en effet à chaque individu non seulement de prendre connaissance des faits, mais aussi de prendre la parole, en adhérant et en contribuant ainsi de manière plus autonome et plus réfléchie aux revendications du groupe. Nous nous intéresserons ici non aux caractéristiques spécifiques du technodiscours des Sentinelles (qui mériterait une étude à part entière), mais à cet «espace hybride» d'action (Castells, 2012, p. 11), à la fois technologique, culturel, idéologique et matériel, qui se déploie entre l'espace urbain et l'espace numérique et dans lequel se construit la signification de la posture des Sentinelles.

Nous mobiliserons aussi partiellement le discours de la presse quotidienne nationale française en nous demandant comment la signification de la posture des Sentinelles, élaborée dans la relation entre discours et mise en scène, est ailleurs saisie, représentée, transformée.

Dans un premier temps, nous étudierons les caractères spécifiques, dits et montrés, de la mise en scène des Sentinelles par rapport à celle des Veilleurs, le groupe avec lequel les Sentinelles reconnaissent un lien de filiation idéologique, ainsi que par rapport à celle du standing man d'Istanbul en 2013, dont la modalité les a directement inspirées.

La complexité de la posture des Sentinelles nous amènera à prendre séparément en compte ses différents aspects - la station verticale et immobile, l'espacement, le silence et l'absence de tout signe distinctif. Il s'agit cependant d'une nécessité déterminée par la démarche d'analyse : en réalité, la signification de cette posture découle évidemment de l'articulation entre ses diverses caractéristiques.

Dans un deuxième temps, nous reviendrons sur ces caractéristiques pour analyser les fonctions dont cette posture se trouve investie lorsqu'elle est montrée sur la place et dite en discours.

Le corpus se compose de tweets, de posts sur le réseau social Facebook, d'extraits de sites internet, publiés par les Sentinelles ou par des énonciateurs sympathisants. La vaste profusion des technoproductions langagières sur ce sujet - en particulier sur Twitter et Facebook -, qui plus est sur une période de plus de deux ans, nous impose cependant, pour commencer, de soumettre le corpus contextuel de référence à des critères sélectifs bien définis.

Le premier est événementiel : seules ont été retenues les productions langagières produites à l'occasion et aux alentours de dates clés. En particulier, ont été examinés les tweets et les posts produits le jour même de la première manifestation des Sentinelles (24 juin 2013), ou les jours ayant immédiatement suivi, ou encore à l'occasion du premier et du deuxième anniversaire de cette manifestation. De même, les dates situées aux alentours du $1^{\text {er }}$ septembre 2013 
ont été prises en considération, lorsque les Sentinelles ont acquis leur nom en abandonnant leur ancienne désignation de Veilleur(s) debout.

L'autre critère est thématique : seuls ont été retenus les tweets, posts et documents numériques portant sur la mise en scène des Sentinelles.

Ces technoproductions langagières sont plurisémiotiques (Paveau, 2013a) : c'est pourquoi, tout en présentant ces productions en dehors de leur environnement numérique complet (en raison notamment de la difficulté de tout reproduire par capture d'écran), nous avons jugé nécessaire, lors de l'analyse du corpus d'étude, de signaler la présence d'images accompagnant certaines de ces productions langagières ainsi que de garder, pour les tweets, la saillance des liens hypertextuels et des hashtags par les caractères gras (mais il manque notamment les avatars). Observons enfin que la présence des hashtags permet la constitution de sous-corpus-tweets thématiques dont la cohérence découle de l'activité métalinguistique et technodiscursive des twitteurs eux-mêmes (Zappavigna, 2012) : en choisissant d'employer un hashtag (\#sentinelles, par exemple), ceux-ci signalent leur «affiliation diffuse » à une communauté ayant des intérêts communs (voir aussi Paveau, 2013a).

Les mêmes critères ont été adoptés pour le corpus de presse (rappelons que, dans notre étude, ce dernier fonctionne comme un sous-corpus contrastif par rapport au corpus «Sentinelles»). Dans ce cas, en puisant dans la base de données Europresse, nous avons retenu les articles publiés par quatre quotidiens nationaux (Le Monde, Libération, Le Figaro, La Croix) au sujet de la posture des Sentinelles depuis le 24 juin 2013 et au cours des mois de juin et juillet 2013 (ainsi qu'à l'occasion des anniversaires, les 24 juin 2014 et 2015) et aux alentours du $1^{\text {er }}$ septembre 2013.

Le corpus d'étude puise dans ce corpus de référence pour proposer l'analyse de certaines de ces productions langagières, organisées en fonction des cheminements de sens dégagés.

\section{La mise en scène des Sentinelles}

Les Sentinelles s'inscrivent dans la mouvance des groupes de contestation nés de LMPT, tels que le Printemps français ou d'autres groupes dont la reconnaissance passe par des signes vestimentaires comme l'écharpe blanche des Mères Veilleuses ou, en polémique avec les Femen, les vestes blanches des Antigones, le torse nu et le masque blanc des Hommen.

\section{Des Veilleurs aux Sentinelles}

Les Sentinelles se sont d'abord appelées «Veilleurs debout» : par cette désignation, une filiation était revendiquée avec les Veilleurs, groupe issu de LMPT 
et organisé par de jeunes animateurs cultivés et fortement marqués par le catholicisme (Tartakowsky, 2014, p.189). Le tout premier Veilleur debout est lui-même un Veilleur.

Les Veilleurs commencent à manifester le soir du 16 avril 2013 par solidarité avec des militants interpellés lors d'une manifestation contre le projet de loi du gouvernement socialiste autorisant le mariage des couples homosexuels. Plus généralement, ils manifestent en faveur d'un programme d' "écologie humaine » qui récuse l'individualisme et le dérèglement de la modernité (Tartakowsky, 2014).

Tout en s'inspirant des principes et des modes de la non-violence qu'ils revendiquent souvent, notamment par référence à Gandhi, les Veilleurs et les Veilleurs debout n'occupent pourtant pas l'espace de la même manière. Alors que les Veilleurs sont assis par terre, les Veilleurs debout choisissent la station immobile et debout; les Veilleurs restent ensemble, les uns à côté des autres, tandis que les Veilleurs debout préconisent l'espacement des manifestants; enfin, les Veilleurs lisent, chantent et exposent des textes sur des banderoles (sans hésiter à s'approprier des auteurs traditionnellement de gauche comme Gramsci), alors que les Veilleurs debout s'imposent le silence et l'absence de tout signe distinctif.

Les Veilleurs debout naissent à la suite de l'arrestation de Nicolas BernardBuss, jeune Veilleur incarcéré le 19 juin 2013 lors d'une manifestation contre le mariage homosexuel. Par solidarité, l'un de ses camarades, médiatisé sous le nom de Jean-Baptiste, manifeste debout devant le ministère de la Justice le 24 juin 2013. L'appropriation de l'espace public en tant que lieu du faire politique passe alors non seulement par la présence de Jean-Baptiste sur la place Vendôme, mais surtout par le rassemblement de voix autour de lui qui se lèvent et circulent à travers les réseaux sociaux et qui participent à la mise en discours de la posture du premier Veilleur debout. Les premiers tweets sont diffusés le jour même, par exemple :

Pierre-Yves @PYves_P24 juin 2013

Il résiste, immobile, dvt le ministère de la justice. N’hésitez pas à le rejoindre \& à prendre le relai! \#onlacherien

نBeabeяtن @berdoeuves 24 juin 2013

Venez soutenir le manifestant immobile. Ministère de la justice. @Les_Veilleurs \#onlr \#PourLesMômes \#veilleurs

Une photographie du jeune manifestant accompagne ces deux tweets : vu de dos, les mains dans les poches, son sac à dos posé entre ses jambes, il fait face au ministère de la Justice. Tel un badge, une petite pancarte écrite à la main et appuyée à ses pieds, derrière lui, porte l'inscription : «veilleurs ». Commence ainsi une forme de manifestation qui ne sera toutefois plus celle des Veilleurs.

Très peu de temps après, la présence de Jean-Baptiste devient un événement discursif officialisé par la création du hashtag \#veilleurdebout : le choix 
de la désignation est déterminé par la posture. Par son pouvoir mimétique, cette désignation fonctionne dès lors comme une catégorisation fondée sur une procédure de reconnaissance perceptive (Conein, 2005). On retrouve, dans les tout premiers tweets du 24 juin, des messages qui, en même temps qu'ils prescrivent d'employer cette nouvelle désignation pour catégoriser et supporter l'individu solitaire posté devant le ministère de la Justice, préconisent aussi la formation, sur place, d'un groupe autour de lui :

نBeabertن @berdoeuves 24 juin 2013

Utilisez le mot \#veilleurdebout pour @jbrristou et tous ceux qui prendront la suite.

Lazarus @EsperanceParis 24 juin 2013

\#veilleurdebout est hashtag pour soutenir @jbrristou debout depuis 4 h devant Min de justice. Qui peut prendre relais tt de suite? \#Veilleurs

L'isolement de Jean-Baptiste ne dure pas longtemps : alors que sa photo et l'annonce de sa présence sur la place Vendôme commencent à circuler dans les réseaux sociaux, d'autres militants le rejoignent. Un deuxième hashtag est alors créé, au pluriel cette fois : \#veilleursdebout. Jean-Baptiste lui-même s'en sert aussitôt dans plusieurs tweets rédigés ce jour-là, dont celui-ci :

JB Tourris @jbrristou 24 juin 2013

\#veilleursdebout veille depuis $5 \mathrm{~h}$ devant ministère de la justice, qui ferme maintenant ses portes.

\#resitance\#ONLR. On reprend bientôt.

Le verbe au singulier, veille, se combine au sujet pluriel Veilleursdebout. Chacun peut désormais être reconnu comme «veilleurs debout», comme représentant d'un groupe dont l'existence est consacrée au niveau discursif par l'acte de désignation, ainsi que par l'affiliation via le hashtag, avant même qu'elle ne soit confirmée par le nombre des manifestants et la durée de leur présence sur la place. Ce processus correspond à ce que dit M. Castells (2012) à propos de la fonction constructive des espaces virtuels de communication permettant la production du sens autour des mouvements sociaux avant même que ceux-ci n'occupent concrètement l'espace public.

Nicolas Bernard-Buss est libéré le 9 juillet 2013. Mais les Veilleurs debout ne cessent pas leur mobilisation : ils demandent désormais l'abrogation de la «loi Taubira» (loi n² 2013-404 du 17 mai 2013) ouvrant le mariage aux couples de personnes du même sexe, tout en s'opposant notamment à la procréation médicalement assistée (PMA) pour les couples homosexuels, à la gestation pour autrui (GPA) pour tous, à l'évocation des questions de genre dans l'éducation publique, à l'euthanasie.

Peu de temps après leur première apparition, les Veilleurs debout changent de nom. De nombreux tweets le signalent, dont notamment celui-ci, qui présente de nouveau la combinaison entre les hashtags nominaux de l'apposition et de l'attribut du sujet, d'une part, et le sujet et le verbe au singulier, de l'autre : 
Vladimir@vladimir651418 31 ago 2013

La veillée étant terminée, \#Veilleurs je deviens \#Sentinelles place Vendôme

Alors que les tweets relaient l'information du changement de nom, la page Facebook est investie d'une fonction explicative, rapide et exhaustive à la fois, quant aux raisons de ce changement. On y apprend que c'est sous l'influence d'autres groupes étrangers - en particulier les Sentinelle in piedi en Italie et les Centinelas de pie en Espagne (à leur tour inspirées par les Veilleurs debout) que le mot sentinelle (qui était déjà, depuis juin 2013, le nom d'un compte Twitter ayant comme avatar l'image du premier Veilleur debout sur la place Vendôme) est adopté le 31 août 2013, lors de l'allocution prononcée à l'issue de la «marche» des Veilleurs. Ce changement permet à la fois d'affirmer une sensibilité culturelle (et de chercher une visibilité médiatique) transnationale tout en marquant une différence par rapport aux Veilleurs :

POURQUOI «Les Sentinelles»?

- par solidarité avec l'Italie et l'Espagne qui partagent le même combat (Sentinelle in piedi, Centinelas de pie)

- pour éviter la confusion entre Veilleurs et Veilleurs Debout, et laisser aux Veilleurs leur spécificité et le rayonnement qu'ils méritent. [...]

\section{QUEL CHANGEMENT?}

> Veiller debout est un mode d'action libre et spontané. Il n'appartient à personne. Tous ceux qui veulent veiller debout le font pour les raisons qui leur appartiennent. ${ }^{1}$

Pour les Sentinelles, il ne s'agit pas de faire bloc (tous ensemble, assis, coude à coude), mais d'affirmer un mode d'action plus individuel, quoique concerté, hérité des Veilleurs debout:

Le veilleur debout ne porte pas de signe distinctif et ne fait pas de récupération, il comprend que chaque action est spontanée et que chacun est libre. ${ }^{2}$

La revendication du caractère singulier et autonome de la manifestation, et surtout l'absence de signes distinctifs, tout en marquant une différence par rapport aux Veilleurs, semblent fonctionner comme la réalisation concrète du «processus d'individuation » qui, selon Danielle Tartakowsky (2014, p. 196197), caractérise les manifestations de droite : chaque manifestant intervient selon sa propre conscience, sans se réclamer d'une tradition, d'une histoire sociale ou politique commune. Cette «an-historicité assumée », ce refus «d'introduire le temps de l'histoire, propre à contrevenir à celui de la nature», serait, selon D. Tartakowsky, l'expression de la défense d'un ordre social considéré

1. https://fr-fr.facebook.com/lessentinelles/posts/607808715932555, 31 août 2013. Tous les liens internet ont été vérifiés le 10 novembre 2015.

2. http://pourkoijeveille.net/pour-comprendre/ce-que-nous-sommes-aujourdhui/charte-desveilleurs-debout. 
comme naturel et immuable (et qui serait représenté, par exemple, par «l'épanouissement de l'enfant au sein de sa famille biologique »)3.

Mais le changement de nom instaure aussi un rapport plus complexe entre les Sentinelles et les Veilleurs debout, entre continuité du mode d'action et effets désignatifs de transformation.

Bien que la désignation sentinelle n'affirme plus de manière transparente le caractère de la station debout, celui-ci demeure un trait distinctif qui accuse la continuité entre les deux mouvements. Les Sentinelles ne cessent de se revendiquer de cette posture, par exemple à l'occasion de leur deuxième anniversaire :

\section{\#2ansDEBOUT!}

Les \#Sentinelles fêtent leur 2 ans de mobilisation le mercredi 24 juin 2015 place Vendôme, dès 19 h 30. Merci de vous inscrire sur l'événement facebook et/ou de le PARTAGER au maximum! \#ONLR. 4

Le hashtag \#2ansDebout transforme l'anniversaire des Sentinelles en événement discursif tout en confirmant que leur reconnaissance se fonde sur la mise en scène de la station debout. Le hashtag \#ONLR (on ne lâche rien) est, quant à lui, un slogan sous forme de sigle largement utilisé par toute LMPT qui, dans sa version non standard - on lâche rien - était d'abord celui du candidat du Front de gauche Jean-Luc Mélenchon à l'élection présidentielle de 2012. Là encore, on assiste à un processus d'appropriation par la droite des symboles de la gauche (rappelons aussi la citation de Gramsci faite par les Veilleurs) ou, selon D. Tartakowsky, au caractère désormais interchangeable de ces symboles dans un processus d'estompage progressif des différences formelles entre manifestations de droite et de gauche (2014, p.194).

En ce qui concerne les effets désignatifs de transformation, on constate que le mot sentinelle semble inscrire le groupe dans une dimension plus conflictuelle. Alors que, pour Veilleurs debout, la mémoire du discours évangélique (la veillée de Jésus dans Mathieu 26, 40-41: «Demeurez ici et veillez avec moi. [...] Veillez et priez afin de ne pas tomber au pouvoir de la tentation ») semblait susciter la représentation d'un groupe caractérisé par une attitude de recueillement attentif, la nouvelle désignation sentinelle contribue, au niveau lexical aussi bien qu'interdiscursif, à une représentation plus belliqueuse (quoique toujours non violente) de l'action de ce groupe. Elle semble porter la mémoire du discours vétéro-testamentaire - «Fils d’homme, je t'établis guetteur pour la maison d’Israël [...]» (Ézéchiel 3, 17)5 - et renvoyer, par sa propre signification lexicographique (le soldat « chargé de faire le guet» et de «protéger un lieu

3. http://pourkoijeveille.net/pour-comprendre/ce-que-nous-sommes-aujourdhui/manifestedes-sentinelles.

4. http://fr-fr.facebook.com/lessentinelles.

5. La Bible, traduction œcuménique, 1988, Paris, Société biblique française et Cerf. 
public », selon Le Petit Robert), à une fonction de défense dans une situation de conflit. Cette représentation peut également surgir du discours lui-même des Sentinelles, lorsqu'elles recourent au répertoire lexical de la guerre (défendre, donner l'alerte, sauver la Cité) pour définir leur fonction :

Les Sentinelles se dressent pour défendre l'Homme et la civilisation. ${ }^{6}$

Les Sentinelles sont des guetteurs, résolus à donner l'alerte pour sauver la Cité [...].7

Une représentation militaire est d'ailleurs déjà entretenue par la pratique des Veilleurs debout (et perpétuée par les Sentinelles, en mode Veilleur debout selon leur manifeste «Attitude») par la disposition spatiale choisie et prescrite :

Sentinelles ne forment pas de groupe compact mais font en sorte d'être espacées les unes des autres de quelques mètres. ${ }^{8}$

Déterminée par le souci de contourner la loi réprimant les attroupements non autorisés, une sorte d'organisation proxémique et de chorégraphie statique se crée ainsi, qui n'est pas sans rappeler la disposition collective des soldats à la parade : la posture debout du soldat acquiert toute sa signification, comme l'observe Georges Vigarello, lorsque chaque corps, chaque posture, sont placés en relation avec les corps et la posture debout des autres soldats. Se dessine ainsi un schéma corporel qui n'est pas celui de la masse amorphe mais de l'orthogonalité : l'incorporation visible et ostentatoire de la rectitude morale se fait par «l'acquisition renforcée de principes d'ordre rigoureux et collectifs» (Vigarello, 2004, p. 56).

Enfin, tant pour les Veilleurs debout que pour les Sentinelles, la mise en scène repose sur le silence observé dans des lieux typiquement politiques et constitutivement liés à la parole. Ce choix inscrit les Veilleurs debout et les Sentinelles dans une forme de manifestation désormais bien connue : la Silent Parade des Afro-Américains à New York en 1917 ou, comme le rappellent Denis Barbet et Jean-Paul Honoré (2013), le silence de la Marche du Sel de Gandhi en 1930 ou celui des «cercles de silence » des frères franciscains de Toulouse depuis 2007 contre les conditions de vie des étrangers placés en centre de rétention. Mais le silence de ces «cercles » se laisse généralement occuper par du discours écrit et affiché (pancartes explicatives, tracts, banderoles), alors que le silence des Veilleurs debout / Sentinelles se donne, en général, comme exclusif : il s'associe en effet à l'absence de tout signe distinctif (y compris linguistique). Ce silence n'échappe pourtant pas au régime du dire. Proclamé

6. http://pourkoijeveille.net/pour-comprendre/ce-que-nous-sommes-aujourdhui/manifestedes-sentinelles/.

7. http://www.lejdv.fr/annonce-creation-les-sentinelles.

8. http://pourkoijeveille.net/attitude-de-la-sentinelle-en-mode-veilleur-debout/. 
comme tel, il est constamment parlé ailleurs, dans et par le discours que les Veilleurs debout / Sentinelles produisent à son propos9. Bien qu'occasionnellement, il est parfois même interrompu au cours même de la mise en scène : par exemple, par la pancarte «veilleurs » du premier manifestant, par des artefacts langagiers comme la paire de chaussures d'un Veilleur debout portant le sigle ONLR photographié et relayé par plusieurs tweets le 1er juillet 2013, ou encore comme la chanson diffusée dans une vidéo sur la mobilisation des Veilleurs debout le matin du 29 juin 2013 , lorsque certains manifestants ont entonné la Strasbourgeoise, chant militaire d'une petite fille de Strasbourg revendiquant fièrement son identité française face à l'ennemi allemand lors de la guerre franco-prussienne de $1870^{10}$.

\section{Le Duranadam et les hommes debout}

Station debout et immobile, espacement, absence de signe distinctif et silence sont donc préconisés et prescrits dans le discours des Sentinelles. Cette performance n'est cependant pas une forme inédite d'action, mais s'inspire de celle du standing man (ou duranadam) de la place Taksim à Istanbul. Le 17 juin 2013, le chorégraphe et artiste Erdem Gündüz est resté debout pendant plusieurs heures, en silence, devant le Centre culturel Atatürk de la place Taksim à Istanbul, pour manifester contre le projet gouvernemental de reconstruction d'une ancienne caserne à la place du Parc Gezi et pour exprimer son indignation après la répression tragique du 31 mai 2013. D’abord inobservé, il est ensuite remarqué et photographié : sa photographie (vu de dos, les mains dans les poches, son sac à dos posé entre ses jambes : c'est bien l'image qui a inspiré la posture du premier Veilleur debout) est très vite relayée par les réseaux sociaux - avec la création du hashtag \#duranadam - selon un processus qui, tout en faisant du duranadam une icône de la résistance non violente contre les exactions du gouvernement turc, le transforme en événement iconographique, discursif et politique. La filiation entre le duranadam et les Veilleurs debout est vite signalée, notamment sur Twitter. Voici un exemple :

Pierre LazaЯ @Pierre_Lazar 24 juin 2013

Suivez@veilleurdebout \#duranadam français

Le processus discursif d'acclimatation du duranadam au contexte français passe ici par la référence au veilleur debout désigné par le mot duranadam et qualifié par son identité nationale (français) ou, ailleurs, par le recours à la traduction française du mot duranadam pour qualifier les Sentinelles, comme dans ce communiqué de presse posté un an après, le 24 juin 2014 :

9. Nous y reviendrons dans le paragraphe «La vertu du silence».

10. https://www.youtube.com/watch?v=AGry2qqhjio. 
Ces courageux «hommes debout» continueront à se mobiliser malgré les tentatives d'intimidation de la Préfecture de Police. ${ }^{11}$

Sans entrer dans l'analyse historique de la contestation turque autour du parc Gezi, soulignons que la similitude de performance entre le duranadam et les Veilleurs debout ne doit pas faire oublier l'opposition entre une manifestation dénonçant la violence répressive d'un pouvoir réactionnaire - le gouvernement turc, comme le souligne Zeynep Gambetti (2014), n'hésitait d'ailleurs pas à mobiliser massivement dans son discours la représentation de la famille traditionnelle comme socle de l'ordre social et comme rempart contre une jeunesse turque en détresse - et une manifestation qui, dénonçant certes un État socialiste jugé répressif, s'oppose à un changement social perçu comme menaçant l'ordre et les valeurs sociales traditionnelles.

Par ailleurs, la mémoire sémantique resurgit parfois pour relier la posture du Veilleur debout à celle du tankman, l'homme inconnu qui a fait face, debout, aux chars de la place Tian'anmen le 5 juin 1989. L'emploi d'un motévénement toponymique (Moirand, 2007) comme «Tian'anmen » ou l'utilisation de la célèbre photo de cet homme devant les chars dans certains tweets permettent alors d'inscrire les Veilleurs debout dans la tradition des manifestants héroïques debout :

Guido.dC@Menfain 25 juin 2013

Souvenez vous de l'homme de Tian'anment, il n'avait que ses deux jambes et son courage \#veilleursdebout \#Vendome

\section{La mise en scène des Sentinelles dans la presse}

En s'inspirant du duranadam, les Veilleurs inscrivent leur posture debout sur la place Vendôme dans les «moyens d'action qui ont déjà leur histoire » (Tilly, 1984, p.103) : ils mobilisent ainsi le répertoire des militantismes non violents ainsi que de ceux qui, récusant le conflit ouvert, visent «à convertir un public à la justesse de la cause défendue [...] sans jamais être agressifs », comme l'explique Lilian Mathieu (2011, p.47). Ces mouvements cherchent traditionnellement la plus large visibilité médiatique en recourant à des formes de «théâtralisation » comme les happenings et les performances.

Or, la presse nationale française peine à parler des Veilleurs debout : les articles consacrés à leur première mobilisation sont tardifs et aucun journal ne traite ni de l'adoption du nom Sentinelles, ni des rassemblements d'anniversaire. Les Sentinelles elles-mêmes entretiennent un rapport conflictuel avec la presse : rappelons, sans pouvoir l'analyser comme elle le mériterait, la critique faite par les Sentinelles de la «désinformation médiatique» dont elles s'esti-

11. https://www.facebook.com/events/1430623770536319/permalink/1437726099826086/. 
ment les victimes. Dans un post publié sur Facebook le 25 juin 2014, elles s’en prennent à un article paru le même jour («Debout et immobile : la nouvelle forme de contestation en vogue») dans Le Figaro, qui est pourtant le journal le plus sensible à leurs revendications. Ce post souligne, correcteur jaune à la main, les nombreuses inexactitudes qui émaillent l'article, notamment celle qui consiste à ne jamais nommer les Sentinelles et à ne parler que des «veilleurs » ${ }^{12}$.

Au cours des mois de juin et juillet 2013 - alors que les tweets et les posts sur Facebook se multiplient à leur sujet -, la presse la plus critique à l'endroit de LMPT ne leur consacre aucun article ni aucune description spécifique (deux articles sont cependant publiés au sujet de la constellation de groupes issus de LMPT, sans aucune description des Veilleurs debout, dans l'édition du 17 juillet 2013 du Monde).

La presse plus sensible aux positions catholiques ne consacre que quelques lignes à la posture des Sentinelles. Parmi les articles de La Croix sur les manifestants issus de LMPT (24 juin 2013, 5, 6, 9, 10, 12, 18 juillet 2013), deux seulement décrivent brièvement la posture des Veilleurs debout. L'un des deux (9 juillet 2013) souligne seulement l'alignement des manifestants; l'autre (même édition) sollicite une représentation non prototypique de cette manifestation en recourant aux formes de la négation portant sur certains aspects (voix, banderoles) typiquement associés au répertoire d'une manifestation :

Postés à quelques mètres les uns des autres, ne se parlant pas entre eux, n'arborant pas de signes distinctifs, ils posent davantage de difficultés aux policiers que les autres manifestants. ${ }^{13}$

Le Figaro - qui est d'ailleurs le seul journal soulignant l'héritage du duranadam chez les Veilleurs debout (2 juillet 2013) - est aussi celui qui parle le plus des Veilleurs debout $(2,4,9,10,12,13,23,24,27$ juillet 2013) en décrivant (quoique rarement) leur posture :

Ils ne scandent aucun slogan, ne déploient aucune banderole, n’arborent aucun signe de ralliement. Ils n'ont l'air de rien comme cela, impassibles, les mains dans les poches, une cigarette aux lèvres ou un livre sous le bras. [...] Ils ne manifestent pas; ils «veillent» tout simplement. Debout. ${ }^{14}$

Ici encore, les négations abondent pour décrire la posture des Veilleurs. La marche, les cris et les slogans typiques des manifestations sont remplacés par l'immobilité et le silence : ces éléments se donnent d'abord en tant qu'absence signifiante (Poyatos, 2002, p.305). La négation porte enfin sur l'action ellemême (ils ne manifestentpas) et précède une rectification - ils «veillent» - : si

12. https://www.facebook.com/lessentinelles/photos/a.608244232555670.1073741835.603529 $186360508 / 773051509408274 /$ ?type=3\&theater.

13. La Croix, «Militants et policiers s'opposent sur le bien-fondé des interpellations », 9 juillet 2013.

14. Le Figaro, «Mariage gay : les anti réinventent la contestation », 2 juillet 2013. 
les guillemets signalent le dire d'autrui, c'est le journaliste lui-même qui souligne par la suite l'évidence de la signification de cette action (tout simplement) ainsi que sa modalité, présentée comme déroutante : debout est en effet placé après le point final, en position isolée, marquée, sollicitant au niveau prédiscursif une opposition avec les modalités plus traditionnelles d'une manifestation (défilés ou sit-in).

Quelques jours plus tard, un autre article se borne à évoquer la pose des Veilleurs debout :

Mardi soir, alors que la trentaine de «veilleurs debout» place Vendôme apprenait la libération de Nicolas, la quasi-totalité d'entre eux, après un soupir de soulagement, reprenait immédiatement la pose. ${ }^{15}$

La pose, comme une sorte de caractère prototypique des Veilleurs debout, leur est ainsi désormais associée : l'évidence dispense de la description. La pose a ses formes qu'il n'est plus utile de rappeler; la pose a aussi sa fonction, sa signification : comme l'affirme le titre même de l'article, il s'agit d'un faire face.

\section{La polémique des Sentinelles}

Le conflit qu'engagent les Sentinelles contre le gouvernement et le «mariage pour tous " s'exprime aussi bien dans leur discours que dans la pose ellemême : exhibée devant les lieux symboliques du pouvoir, celle-ci se charge d'une fonction polémique (quoique physiquement non violente) au sens que Ruth Amossy donne à cette notion (2014, p. 51) : "antagonisme des opinions », "opposition de discours» autour d'une question d'actualité impliquant des enjeux de société. Cette pose met en scène, montre la dichotomie, "dans un face-à-face où elles [les parties] campent sur des positions inconciliables» (Amossy, 2014, p. 57). La fonction polémique de cette posture est souvent dite en discours (ici, par exemple, par la mobilisation d'un paradigme d'opposition : désaccord, défi) :

La Sentinelle exprime spontanément son opinion ou son désaccord politique par sa posture, statique et pacifique [...]. ${ }^{16}$

Les Sentinelles se tiennent debout, immobiles, espacées les unes des autres, sans slogan ni signe distinctif face à des lieux symboliques, tel un défi au pouvoir en place. ${ }^{17}$

15. Le Figaro, "Les "veilleurs" restent debout "face aux autres injustices" ", 10 juillet 2013.

16. http://pourkoijeveille.net/attitude-de-la-sentinelle-en-mode-veilleur-debout/.

17. Les Sentinelles Officiel, https://www.facebook.com/events/1430623770536319/ (communiqué de presse) 
La posture est aussi, pour les Sentinelles, un acte de résistance non violente contre les décisions gouvernementales qu'elles stigmatisent : destruction, dénaturation, atteinte, endoctrinement signifient, dans leur argumentation, la décomposition violente de l'ordre «naturel» :

Nous témoignons à notre façon de notre Résistance inébranlable face aux tentatives de destruction de la Famille Père/Mère/Enfant - socle de notre civilisation - par la dénaturation du Mariage, l'atteinte à la dignité humaine (euthanasie, marchandisation du corps avec la PMA ou la GPA, manipulation de l'embryon humain...) ou encore l'endoctrinement des enfants à l'idéologie du Genre dès la petite enfance. ${ }^{18}$

Souvenons-nous de la nouvelle «Deux amis» de Maupassant. Comme l'observait Algirdas Julien Greimas à propos des deux pêcheurs qui restent debout et immobiles face à l'officier prussien qui les interroge, la posture debout n'est pas anodine : "La station "debout" [...] prend des allures d'un faire somatique signifiant, d'un "faire face” [...] ni l'“immobilité" ni la station "debout” ne sont ici des attitudes corporelles “naturelles” » (Greimas, 1976, p. 212). La posture debout est donc polémique : c'est un faire face - faire face à l'ennemi prussien, aux tanks de Tian'anmen, à la répression du parc Gezi, à la menace représentée par ce que les Sentinelles considèrent comme le symbole des abus du pouvoir, le ministère de l’Injustice, selon leur propre expression.

\section{La vertu du silence}

Le silence qui caractérise la station debout dans «Deux amis » est un silence qui montre le choix de ne pas dire et supporte le poids d'une parole consciemment retenue. Le silence des Sentinelles, en revanche, est un silence qui, toujours pointé par leur discours, vise à montrer qu'il est à l'œuvre en tant qu'absence proclamée de parole. Dans le tweet suivant, par exemple, c'est en particulier un hashtag adjectival qui se charge de dire ce silence caractéristique :

Les Sentinelles @Sentinelles_fr 18 juillet 2013

En \#resitancepacifique le \#veilleurdebout reste \#silencieux parle pas chante pas joue pas avec crs. Son silence est sa force irrépressible.

"L'éloquence n'est pas seulement affaire de mots mais aussi affaire de silences qui en disent long » (Le Breton, 1997, p. 79) : le silence des Sentinelles est censé dire beaucoup, mais il semble ne pas se suffire à lui-même puisqu'il est constamment signalé, invoqué, expliqué par leur propre discours (l'existence et le nom du site "pourkoijeveille», géré par des Veilleurs et des Veilleurs debout, suffisent d'ailleurs sans doute à montrer l'urgence explicative du discours, notamment à propos du silence). Nombreux sont les moments où

18. https://www.facebook.com/events/1430623770536319/. 
les Sentinelles s'efforcent d'éclairer les différents sens de leur silence, dont par exemple :

[...] nous nous indignons contre l'injustice et les mensonges de nos dirigeants, en les toisant pacifiquement devant des lieux symboliques et des institutions de l’État. 19

Le sentiment d'indignation dont les Sentinelles peuvent charger leur silence semble confirmer ce qu'observe M. Castells (2012) à propos des mouvements sociaux : ceux-ci, tels Occupy Wall Street ou les indignados d'Espagne, naissent sous l'impulsion d'une expérience émotionnelle individuelle qui cherche à être partagée. Mais le silence dont il est question ici ne se donne pourtant pas sous la forme de la spontanéité : explicitement dit $^{20}$ et clairement affiché en discours, il passe, selon une opposition encore soulignée par Christian Plantin (2011), du niveau émotionnel au niveau émotif, réfléchi, stratégique. Pour les Sentinelles, ce niveau se déploie à la fois en discours et dans la rue. Au lieu de crier leur indignation, elles se taisent en la disant en discours :

Parce qu'un long silence vaut parfois mieux qu'un grand discours, nous nous relayons, debout face à des lieux de pouvoirs [...].21

Ce silence ne cède pas à l'émotion représentée par le stéréotype sémiologique des cris, ni n'affiche d'ailleurs un état an-émotionnel, mais cherche à se montrer vertueux et persuasif par sa contenance émotive. La vertu discursive (Paveau, 2013b) qui consiste à faire preuve de tempérance au niveau langagier, longuement entretenue par la tradition rhétorique et maintenue au niveau prédiscursif, se trouve dans le silence qui met en scène la résistance indignée aussi bien que dans les mots qui le manifestent.

\section{Entre mise en volume et mise en mots}

En distinguant le geste de l'agir et du faire (selon une opposition proposée par Giorgio Agamben), les éditeurs de l'ouvrage Des gestes en histoire considèrent que «le geste ne peut se réduire ni à une manière de produire, ni à une manière d'agir, mais doit être envisagé en tant qu'il symbolise la volonté d'assumer et de supporter un ensemble de valeurs, une certaine vision du monde » (Ambroise-Rendu et al., 2006, p.13). En ce sens, par les différentes fonctions dont elle se charge, toutes dites et imbriquées les unes dans les autres dans cette mise en scène complexe (polémique du corps qui fait face, résistance de

19. Les Sentinelles: https://www.facebook.com/events/1430623770536319/ (page Facebook officielle).

20. Pour une étude sur l'émotion dite et l'émotion montrée, voir Jacquin, Micheli, 2013.

21. Les Sentinelles, page Facebook officielle (citée supra). 
la station debout, autonomie des manifestants qui n'affichent aucun signe distinctif, indignation, notamment, du silence), la posture des Sentinelles peut être considérée comme un geste de vigilance et d'opposition vis-à-vis de ce qu'elles considèrent comme les dérives de la modernité (dont le mariage des couples homosexuels).

Ce geste, comme nous avons essayé de le montrer, n'est pas l'épiphénomène corporel d'un discours qui interviendrait pour l'éclairer, mais correspond plutôt à une "mise en volume» des corps qui se combine constitutivement à une «mise en discours» des manifestants numériques.

En cela, la modalité par laquelle les Sentinelles échangent, s'organisent et se mobilisent rejoint celle, analysée par M. Castells (2012), des plus récents mouvements contestataires (Occupy Wall Street, les indignados, le Printemps arabe), au-delà des traditionnelles oppositions droite-gauche. Le «processus d'individuation » par lequel chacun intervient de manière singulière, autonome, ne caractérise finalement pas seulement les manifestations de droite (qui peuvent pourtant certes se distinguer par leur «an-historicité » idéologique), mais semble commun à tous les mouvements sociaux récents, lorsque ce processus est contrecarré par l'affiliation des individus à des réseaux culturels communs (Farro, 2014, p. 23). Se créent ainsi des espaces hybrides d'action et de production de sens dont l'architecture est, aujourd'hui, celle de l'espace urbain mais aussi celle, numérique, technodiscursive, des sites et surtout des réseaux sociaux sur l'Internet. Consolidés par des revendications idéologiques (politiques et religieuses) encore pugnaces, ces espaces ont contribué à la visibilité, à la structuration, à la signification des premières performances des Veilleurs debout (malgré une presse parfois inattentive) et ne cessent, pour le moment, de cimenter la continuité de cette mobilisation.

\section{Références}

Ambroise-Rendu Anne-Claude, D’Almeida Fabrice, Edelman Nicole, 2006, «Introduction", Des gestes en histoire. Formes et significations des gestualités médicale, guerrière et politique, A.-C. Ambroise-Rendu, F. D’Almeida, N. Edelman éd., Paris, Seli Arslan, p. 9-17.

Amossy Ruth, 2014, Apologie de la polémique, Paris, PUF.

Barbet Denis, Honoré Jean-Paul, 2013, "Ce que se taire veut dire. Expressions et usages politiques du silence ", Mots. Les langages du politique, n 103, p. 7-21.

CARÉnInI André, 1991, "La symbolique manuelle», Histoire des mœurs, t.II, J. Poirier éd., Paris, Gallimard, p.75-162.

CASTELLS Manuel, 2012, Networks of outrage and hope, Cambridge, Polity.

CONEIN Bernard, 2005, Les sens sociaux, Paris, Economica.

FARro L. Antimo, 2014, «A new Era for Collective Movements: the Subjectivization of 
Collective Action», Reimagining Social Mouvements. From Collectives to Individuals, A. Farro, H. Lustigier-Thaler éd., Surrey, Ashgate, p. 15-34.

GambetTI Zeynep, 2014, "Occupy Gezi as Politics of the Body ", The making of a protest movement in Turkey, U. Özkirimli éd., Basingstoke, Palgrave Macmillan, p. 88-102.

Greimas Algirdas Julien, 1976, Maupassant. La sémiotique du texte : exercices pratiques, Paris, Seuil.

Guilhaumou Jacques, 2006, Discours et événement, Besançon, Presses universitaires de Franche-Comté.

JACQUIN Jérôme, MICHELI Raphaël, 2013, «Dire et montrer qui on est et ce que l'on ressent. Une étude des modes de sémiotisation de l'identité et de l'émotion », Dire/Montrer. Au cœur du sens, H. De Chanay, M. Colas-Blaise, O. Le Guern éd., Chambéry, Université de Savoie, p. 67-92.

KÆCHLIN Bernard, 1991, "La réalité gestuelle des sociétés humaines», Histoire des mœurs, t. II, J. Poirier éd., Paris, Gallimard, p.163-245.

Le Breton David, 1997, Du Silence, Paris, Métailié.

Londe Danielle, Moirand Sophie, Reboul-Touré Sandrine, Reg gIAnI Licia, 2013, « LeS sens de l'événement», Dire l'événement. Langage, mémoire société, D. Londei, S. Moirand, S. Reboul-Touré, L. Reggiani éd., Paris, Presses de la Sorbonne Nouvelle, p.11-20.

Mathieu Lilian, 2011, La démocratie protestataire, Paris, Presses de Sciences Po.

MoIRAND Sophie, 2007, Les discours de la presse quotidienne, Paris, PUF.

Paveau Marie-Anne, 2013a, «Technodiscursivités natives sur Twitter. Une écologie du discours numérique», Epistémè (revue internationale de sciences humaines et sociales appliquées, Séoul), nº 9, p.139-176, «hal-00859064〉.

- 2013b, Langage et morale, Limoges, Lambert-Lucas.

Plantin Christian, 2011, Les bonnes raisons des émotions, Berne, Peter Lang.

Poyatos Fernando, 2002, Nonverbal Communication Across Disciplines, Amsterdam, John Benjamin Publishing, vol.II.

TARTAKOWS KY Danielle, 2014, Les droites et la rue, Paris, La Découverte.

TilLy Charles, 1984, "Les origines du répertoire de l'action collective contemporaine en France et en Angleterre », Vingtième siècle, nº 4, p. 89-108.

VIGARELLO Georges, 2004, Le corps redressé. Histoire d'un pouvoir pédagogique, Paris, Armand Colin (nouvelle édition avec postface).

Zappavigna Michele, 2012, Discourse of Twitter and Social Media, Londres, New York, Continuum. 